\title{
Erratum: On representing chemical environments [Phys. Rev. B 87, 184115 (2013)]
}

Albert P. Bartók, Risi Kondor, and Gábor Csányi

(Received 14 June 2017; published 17 July 2017)

DOI: 10.1103/PhysRevB.96.019902

In Sec. III G the definition of the angular Fourier series (AFS) should read as

$$
\mathrm{AFS}_{n, l}=\sum_{i, i^{\prime}>i} g_{n}\left(r_{i}\right) g_{n}\left(r_{i^{\prime}}\right) \cos \left(l \theta_{i i^{\prime}}\right)
$$

In Sec. IV the definition of the overlap $S(\hat{R})$ (top right on p. 10) is missing the $r^{2}$ term in the radial part of the integral,

$$
\begin{aligned}
S(\hat{R}) & =S\left(\rho, \hat{R} \rho^{\prime}\right)=\int d \mathbf{r} \rho(\mathbf{r}) \rho^{\prime}(\hat{R} \mathbf{r})=\sum_{i, i^{\prime}} \sum_{\substack{l, m \\
l^{\prime}, m^{\prime}, m^{\prime \prime}}} D_{m^{\prime} m^{\prime \prime}}^{l^{\prime}}(\hat{R}) \int d r r^{2} c_{l m}^{i *}(r) c_{l^{\prime} m^{\prime}}^{i^{\prime}}(r) \int d \hat{\mathbf{r}} Y_{l m}^{*}(\hat{\mathbf{r}}) Y_{l^{\prime} m^{\prime \prime}}(\hat{\mathbf{r}}) \\
& =\sum_{i, i^{\prime}} \sum_{l, m, m^{\prime}} \tilde{I}_{m m^{\prime}}^{l}\left(\alpha, r_{i}, r_{i^{\prime}}\right) D_{m m^{\prime}}^{l}(\hat{R})=\sum_{l, m, m^{\prime}} I_{m m^{\prime}}^{l} D_{m m^{\prime}}^{l}(\hat{R}) .
\end{aligned}
$$

We note that the orthogonality requirement for the radial basis functions (top left on p. 11) also should include the $r^{2}$ term in the integral,

$$
\int d r r^{2} g_{n}(r) g_{n^{\prime}}(r)=\delta_{n n^{\prime}}
$$

In our numerical calculations the integrals were evaluated correctly.

The index of the second spherical harmonic in Eq. (33) was not typed correctly, and the constant prefactor was missing a $\left(\frac{\pi}{2 \alpha}\right)^{\frac{3}{2}}$ term,

$$
\tilde{I}_{m m^{\prime}}^{l}\left(\alpha, r_{i}, r_{i^{\prime}}\right)=\sqrt{\frac{2 \pi^{5}}{\alpha^{3}}} \exp \left[-\alpha\left(r_{i}^{2}+r_{i^{\prime}}^{2}\right) / 2\right] \iota_{l}\left(\alpha r_{i} r_{i^{\prime}}\right) Y_{l m}\left(\hat{\mathbf{r}}_{i}\right) Y_{l m^{\prime}}^{*}\left(\hat{\mathbf{r}}_{i^{\prime}}\right) .
$$

The prefactor is not significant because it is canceled when the kernel is normalized, see Eq. (36).

The final result in Eq. (34) is missing a $\frac{8 \pi^{2}}{2 l+1}$ term, which is due to the normalization of the Wigner $D$ matrices,

$$
\int d \hat{R}\left[D_{m m^{\prime}}^{l}(\hat{R})\right]^{*} D_{\mu \mu^{\prime}}^{\lambda}(\hat{R})=\frac{8 \pi^{2}}{2 l+1} \delta_{l \lambda} \delta_{m \mu} \delta_{m^{\prime} \mu^{\prime}},
$$

resulting in the revised equation,

$$
k\left(\rho, \rho^{\prime}\right)=\int d \hat{R} S^{*}(\hat{R}) S(\hat{R})=\sum_{\substack{l, m, m^{\prime} \\ \lambda, \mu, \mu^{\prime}}}\left(I_{m m^{\prime}}^{l}\right)^{*} I_{\mu \mu^{\prime}}^{\lambda} \int d \hat{R}\left[D_{m m^{\prime}}^{l}(\hat{R})\right]^{*} D_{\mu \mu^{\prime}}^{\lambda}(\hat{R})=\sum_{l, m, m^{\prime}} \frac{8 \pi^{2}}{2 l+1}\left(I_{m m^{\prime}}^{l}\right)^{*} I_{m m^{\prime}}^{l},
$$

and changing Eq. (39),

$$
k\left(\rho, \rho^{\prime}\right)=\sum_{n, n^{\prime}, l, m, m^{\prime}} \frac{8 \pi^{2}}{2 l+1} c_{n l m}\left(c_{n l m^{\prime}}^{\prime}\right)^{*}\left(c_{n^{\prime} l m}\right)^{*} c_{n^{\prime} l m^{\prime}}^{\prime} \equiv \sum_{n, n^{\prime}, l} \frac{8 \pi^{2}}{2 l+1} p_{n n^{\prime} l} p_{n n^{\prime} l}^{\prime} .
$$

The $2 l+1$ factor in the denominator does not change the rotational invariance of the smooth overlap of atomic positions (SOAP) kernel, but it somewhat changes its smoothness properties by weighing down the higher angular momentum terms. We carefully tested our reconstruction experiments and obtained the same results, whereas our interatomic potential fits improved.

Similarly, due to the relation,

$$
\int d \hat{R}\left[D_{m m^{\prime}}^{l}(\hat{R})\right]^{*} D_{m_{1} m_{1}^{\prime}}^{l_{1}}(\hat{R}) D_{m_{2} m_{2}^{\prime}}^{l_{2}}(\hat{R})=\frac{8 \pi^{2}}{2 l+1} C_{l_{1} m_{1} l_{2} m_{2}}^{l m} C_{l_{1} m_{1}^{\prime} l_{2} m_{2}^{\prime}}^{l m^{\prime}},
$$

the $\frac{8 \pi^{2}}{2 l+1}$ factor was missing from Eqs. (35) and (41),

$$
k\left(\rho, \rho^{\prime}\right)=\sum \frac{8 \pi^{2}}{2 l+1} I_{m_{1} m_{1}^{\prime}}^{l_{1}} I_{m_{2} m_{2}^{\prime}}^{l_{2}} I_{m m^{\prime}}^{l} C_{l_{1} m_{1} l_{2} m_{2}}^{l m} C_{l_{1} m_{1}^{\prime} l_{2} m_{2}^{\prime}}^{l m^{\prime}}=\sum \frac{8 \pi^{2}}{2 l+1} b_{n_{1} n_{2} n l l_{1} l_{2}} b_{n_{1} n_{2} n l l_{1} l_{2}}^{\prime} .
$$


We note that $\zeta$, used in Eq. (36) as a hyperparameter of the SOAP kernel, is not restricted to integers, therefore any positive real number may be used.

In the caption of Fig. 7, the value of the kernel should be positive, i.e., $K\left(\rho, \rho^{\prime}\right)=0.842735$.

We would like to clarify that in our reconstruction experiments we used the eight-norm of the expression of $d_{\text {ref }}$ in Eq. (7). We define the eight-norm as

$$
\|\mathbf{x}\|_{8}=\sqrt[8]{\sum_{i} x_{i}^{8}} .
$$

This also implies that the units of $d_{\text {ref }}$ in the text are in angstroms.

In our reconstruction experiments we used the truncation of $j_{\max }=5$ in the case of the $\mathrm{SO}(4)$ bispectrum.

We would like to thank D. Karls and M. Ceriotti for carefully reading the article and pointing out these mistakes. 